\title{
Tradução e adaptação transcultural da versão brasileira da Fear of Negative Evaluation Scale (FNE)
}

\author{
Translation and cross-cultural adaptation of the Brazilian Portuguese version of the Fear of \\ Negative Evaluation Scale (FNE)
}

\author{
Adriana Cardoso de Oliveira e Silva ${ }^{1}$, Antonio Egidio Nardi ${ }^{2}$ \\ ${ }^{1}$ Pós-doutoranda, Programa de Pós-Graduação em Psiquiatria e Saúde Mental, Instituto de Psiquiatria, Faculdade de Medicina, Universidade Federal do Rio de Janeiro (UFRJ), \\ Rio de Janeiro, RJ. Doutora, Psicologia, Instituto de Psicologia, UFRJ. Pesquisadora, Laboratório de Pânico e Respiração, Instituto de Psiquiatria, Faculdade de Medicina, \\ UFRJ. Instituto Nacional de Ciência e Tecnologia (INCT) - Translational Medicine. ${ }^{2}$ Livre-docente, Programa de Pós-Graduação em Psiquiatria e Saúde Mental, Instituto de \\ Psiquiatria, Faculdade de Medicina, UFRJ. Professor associado, Programa de Pós-Graduação em Psiquiatria e Saúde Mental, Instituto de Psiquiatria, Faculdade de Medicina, \\ UFRJ. Coordenador, Laboratório de Pânico e Respiração. INCT - Translational Medicine. \\ O trabalho foi desenvolvido no Laboratório de Pânico e Respiração, Instituto de Psiquiatria, Faculdade de Medicina, UFRJ.
}

\section{Resumo}

Introdução: A Fear of Negative Evaluation Scale (FNE) apresenta como proposta avaliar o medo referente a avaliações sociais em população adulta. Sua adaptação transcultural apresenta-se como primeiro passo para futuras comparações relativas ao construto medido pelo instrumento em diferentes amostras. O presente estudo tem como objetivo a adaptação semântica da FNE para o português do Brasil.

Método: Processo realizado por meio de duas traduções e retrotraduções elaboradas por avaliadores independentes e sem conhecimentos prévios da escala a ser traduzida; elaboração de versão sintética realizada conjuntamente por dois diferentes especialistas bilíngues em saúde mental; posterior aplicação em grupo experimental para avaliação dos itens formados na versão sintética, buscando melhor adequação deles à população à qual a escala se destina.

Resultados: Para cada um dos 30 itens do instrumento são apresentadas as quatro etapas do processo. A utilização de duas traduções e retrotraduções permitiu realizar uma discussão mais ampla para a construção da versão sintética. A participação de especialistas em saúde mental colaborou para a adequação dos termos utilizados em relação ao construto medido. Pela aplicação experimental foi possível identificar pontos de potencial dificuldade para o entendimento de itens específicos por determinados estratos da população, bem como realizar ajustes nesses itens posteriormente.

Conclusão: Por esse método foi possível elaborar a versão para o português do Brasil da FNE.

Descritores: Escalas, psicometria, ansiedade, transtornos de ansiedade.

\begin{abstract}
Introduction: The Fear of Negative Evaluation Scale (FNE) is aimed at evaluating the fear of social evaluations in adults. Its cross-cultural adaptation is the first step for future comparisons regarding the construct measured by the instrument in different populations. The objective of the present study is to semantically adapt the FNE to Brazilian Portuguese.

Method: The process consisted of 1) two translations and back-translations carried out by independent evaluators without any prior knowledge of the scale to be translated; 2) development of a brief version by two different bilingual experts in mental health; 3) subsequent administration to an experimental group to evaluate the items in the brief version, seeking to better match the population to which the scale is intended.

Results: The four stages of the process are shown for each one of the 30 items of the instrument. The use of two translations and back-translations allowed a broader discussion about the design of the brief version. The participation of experts in mental health contributed to the appropriateness of the terms used for the construct measured. The experimental administration of the instrument allowed the identification of potential difficulties regarding the clarity of specific items in some of the population samples and subsequent performance of adjustments.
\end{abstract}

Conclusion: We were able to elaborate the Brazilian Portuguese version of the FNE by means of this methodology.

Keywords: Scales, psychometry, anxiety, anxiety disorders.

\section{Correspondência:}

Adriana Cardoso de Oliveira e Silva, Universidade Federal do Rio de Janeiro, Instituto de Psiquiatria, Laboratório de Pânico e Respiração, Rua Visconde de Pirajá, 407/702, CEP 22410-003, Rio de Janeiro, RJ. Tel.: (21) 2521.6147. Fax: (21) 2523.6839. E-mail: adrianacardosorj@yahoo.com.br

Trabalho realizado no Laboratório de Pânico e Respiração, Instituto de Psiquiatria, Universidade Federal do Rio de Janeiro (UFRJ), Rio de Janeiro, RJ, e no Instituto Nacional de Ciência e Tecnologia (INCT) - Translational Medicine.

Suporte financeiro: Conselho Nacional de Desenvolvimento Científico e Tecnológico (CNPq).

Não foram declarados conflitos de interesse associados à publicação deste artigo.

Copyright (C) Revista de Psiquiatria do Rio Grande do Sul - APRS

Recebido em 07/05/2009. Aceito em 16/07/2009. 


\section{Introdução}

Desenvolvida por Watson \& Friend ${ }^{1}$, a Fear of Negative Evaluation Scale (FNE) apresenta como proposta avaliar o medo referente a avaliações sociais na população adulta. Composta por 30 itens de questões de verdadeiro ou falso, a aplicação desta escala pode ser feita de forma individual, coletiva ou autoaplicada.

Ela atua de forma complementar à Social Avoidance and Distress Scale (SADS), já traduzida e adaptada para o português do Brasil ${ }^{2}$ e composta por 28 itens, também de questões de verdadeiro ou falso, sendo, junto com esta, as duas escalas desenvolvidas para o uso conjunto na mensuração de aspectos ligados à ansiedade social.

Em sua construção, a FNE apresenta itens invertidos para o construto medido, sendo consideradas como respostas padrão "verdadeiro" nos itens $2,3,5,7,9,11,13,14,17,19$, 20, 22, 24, 25, 28, 29 e 30, e "falso" nos itens 1, 4, 6, 8, 10, $12,15,16,18,21,23,26$ e 27.

Devido aos seus baixos custo e tempo de aplicação (em torno de 10 minutos cada), somados à facilidade que implica serem autoaplicáveis, essas escalas mostram-se como opções interessantes para realizar uma avaliação rápida e eficiente, tanto no ambiente em que o sujeito está inserido em seu cotidiano, dessa forma ajustando-se bem à coleta de dados tanto em situações de pesquisa social, quanto no contexto clínico e mesmo hospitalar.

A ausência de marcadores biológicos e de outros parâmetros de medida objetivos para a identificação e avaliação dos transtornos psiquiátricos ${ }^{3}$ colabora para que ocorra uma maior procura por instrumentos psicométricos que, devido a suas características, acabam por preencher essa lacuna, cumprindo importante função.

O crescimento da ênfase outorgada aos métodos baseados em evidências, também na área de saúde mental ${ }^{4-6}$, tem gerado cada vez mais a necessidade de realizar quantificações nas pesquisas em psiquiatria e psicologia, o que também aumenta a busca por instrumentos estruturados que possam fornecer dados obtidos mediante um método padronizado e que ofereçam garantia de validade e fidedignidade para os resultados das referidas pesquisas.

Os estudos dos parâmetros psicométricos da FNE original $^{1}$, realizados com estudantes universitários, demonstraram correlação com medidas de ansiedade, medo de avaliação social e locus de controle, consistência interna de 0,94, avaliada pelo coeficiente Kuder-Richardson (KR20), e fidedignidade de 0,94, aferida por meio de teste-reteste com intervalo de 1 mês.

Ao mesmo tempo, o uso de instrumentos de medida focados em conjuntos específicos de sinais e sintomas tem auxiliado em processos terapêuticos que apresentam em suas propostas metodológicas o treino de reconhecimento pelo paciente desses sinais e sintomas em si próprio e sua posterior automonitoração.

Devemos considerar, ainda, que abordagens psicoterápicas mais recentes, como a terapia cognitivo-comportamental, utilizam escalas para acompanhamento do progresso do paciente ao longo do processo terapêutico, servindo elas, nesses casos, não apenas para quantificar aquilo que se destinam a medir, mas também como guia para o terapeuta em relação a quais procedimentos adotados estão gerando melhor resposta em termos de efetividade terapêutica.

No caso específico dos transtornos de ansiedade, temos notado crescente produção, tanto na elaboração de novos instrumentos ${ }^{7,8}$ quanto na tradução e adaptação semântica para nossa língua de escalas já usadas em outros idiomas ${ }^{2,9-12}$, assim como na realização de estudos voltados para a análise de seus parâmetros psicométricos ${ }^{13-17}$ e na busca pelo estabelecimento de normas para a população brasileira ${ }^{13,14}$.

A tradução e adaptação de instrumentos amplamente utilizados internacionalmente apresentam como vantagem possibilitar a comparação de resultados obtidos com populações de diferentes países e, com isso, gerar dados transculturais $^{18}$.

O medo da avaliação negativa por outros, assim como o de ser julgado desfavoravelmente em situações sociais ou humilhado publicamente caracterizam a fobia social ${ }^{19}$. A ansiedade gerada, junto com seus consequentes sinais e sintomas, pode levar a comportamentos de evitação de ambientes ou situações que envolvam relações interpessoais, prejudicando o funcionamento global do sujeito e comprometendo sua qualidade de vida. A FNE, tendo como foco o medo da avaliação negativa, apresenta-se como um instrumento de grande utilidade para trabalhos relacionados a esse quadro específico.

Este estudo apresenta como proposta a tradução e adaptação semântica da FNE para a população brasileira, abrindo a possibilidade de futuros trabalhos de validação e aferição de fidedignidade do instrumento produzido para essa população.

\section{Metodologia}

O processo de tradução e adaptação semântica da FNE adotado neste estudo, semelhante ao realizado na adaptação da SADS, sua escala complementar, foi baseado na proposta de Herdman et al. ${ }^{20}$. Nesse modelo são seguidas quatro etapas: tradução, retrotradução, análise de equivalência semântica e aplicação experimental.

Dando início ao processo de tradução e retrotradução ${ }^{21,22}$, foi entregue uma versão original da escala a dois tradutores independentes (T1 e T2), fluentes na língua do instrumento original, de naturalidade brasileira, com conhecimentos de psiquiatria e saúde mental e sem qualquer contato prévio com a escala apresentada. Posteriormente, as questões elaboradas por T1 e T2 foram entregues a outros dois tradutores para elaboração das retrotraduções (R1 e R2).

$\mathrm{O}$ material decorrente desse processo foi analisado por dois profissionais especialistas na área de saúde mental e psiquiatria. Foram comparadas as frases geradas nas retrotraduções com o proposto no instrumento original para verificar a equivalência entre os textos. 
Para elaborar a versão sintética foram realizadas comparações entre as propostas das duas traduções e a do texto original para cada um dos itens. Enquanto alguns deles foram definidos basicamente por uma das traduções apresentadas, outros surgiram de uma combinação entre elas, podendo ou não haver alterações realizadas pelos especialistas para uma melhor adequação das formulações. Foi enfatizada a equivalência semântica em detrimento da literal ${ }^{12}$.

Concluída a versão sintética, passamos à etapa de aplicação experimental do instrumento para verificar sua adequação à população alvo. Um ponto importante nessa fase é a identificação de eventuais dificuldades no entendimento dos itens pelos respondentes, uma vez que a sua não compreensão não deve influenciar a resposta do sujeito por não ser esse $o$ atributo a ser mensurado ${ }^{23}$.

Participaram dessa fase do processo 27 voluntários, todos adultos, de ambos os sexos e com diferentes níveis de escolaridade, segundo a seguinte distribuição: nível fundamental: seis feminino, um masculino, sete total; nível médio: quatro feminino, um masculino, cinco total; nível superior: nove feminino, seis masculino, 15 total.

Uma das críticas aos trabalhos sobre instrumentos de medida para transtornos de ansiedade social refere-se ao fato de serem realizados poucos estudos com amostras não clínicas, o que colocaria em questão a efetividade desses instrumentos para rastreamento na população geral ${ }^{24}$. A fim de evitar esse problema, desde a fase de tradução e adaptação do instrumento, optamos por trabalhar com participantes que preenchessem como critério de inclusão não apresentarem transtornos mentais.
Todos os sujeitos voluntários, inicialmente 30 , foram avaliados quanto à presença de transtornos psiquiátricos por meio do Mini International Neuropsychiatric Interview (MINI) versão 5.0.0 ${ }^{25}$, tendo sido descartada a presença de transtornos mentais nos 27 sujeitos que participaram da aplicação.

A aplicação experimental foi composta por três etapas. Os participantes responderam à escala na modalidade autoaplicada. Após a conclusão desta etapa, foi verificada pelo pesquisador a omissão de respostas e registrados os itens em que isso tinha ocorrido para verificação de causa.

Em seguida, foi realizado um questionamento quanto ao sentido dos enunciados, segundo a compreensão dos próprios respondentes, para verificar o real entendimento do sentido das frases por eles. Finalmente, os participantes foram convidados a fornecerem sugestões que pudessem facilitar o entendimento do instrumento ou aprimorá-lo.

Com base nos dados colhidos na aplicação experimental, os enunciados da versão sintética foram revistos, buscando-se solucionar problemas que tenham ocorrido na aplicação por não entendimento ou confusões quanto ao significado dos itens. Foi então constituída a versão final para a FNE em português do Brasil.

\section{Resultados}

A Tabela 1 apresenta as questões do instrumento original, em língua inglesa, as traduções realizadas pelos dois tradutores independentes convidados (T1 e T2) para o português do Brasil, as retrotraduções (R1 e R2) e a versão sintética, elaborada com base nesse processo por profissionais especialistas em saúde mental.

Tabela 1 - Fear of Negative Evaluation Scale (FNE)

\begin{tabular}{|c|c|c|c|}
\hline Original & Tradução & Retrotradução & Versão sintética \\
\hline $\begin{array}{l}\text { 1. I rarely worry about seeming } \\
\text { foolish to others. }\end{array}$ & $\begin{array}{l}\text { T1: Eu raramente me preocupo se } \\
\text { pareço tolo para os outros } \\
\text { T2: Eu raramente me preocupo em } \\
\text { parecer tolo aos outros. }\end{array}$ & $\begin{array}{l}\text { R1: I rarely care if I look stupid/ri- } \\
\text { diculous to the others. } \\
\text { R2: I rarely am concerned to look } \\
\text { foolish to anothers. }\end{array}$ & $\begin{array}{l}\text { Raramente me preocupo em parecer } \\
\text { tolo aos outros }\end{array}$ \\
\hline $\begin{array}{l}\text { 2. I worry about what people will } \\
\text { think of me even when I know } \\
\text { it doesn't make any difference. }\end{array}$ & $\begin{array}{l}\text { T1: Eu me preocupo com o que } \\
\text { as pessoas pensam a meu respeito } \\
\text { quando sei que isso não faz } \\
\text { diferença alguma. } \\
\text { T2: Eu me preocupo com o que as } \\
\text { pessoas pensarão de mim mesmo } \\
\text { quando eu sei que isso não faz a } \\
\text { menor diferença }\end{array}$ & $\begin{array}{l}\text { R1: I care about what people think } \\
\text { of me even when I know this } \\
\text { doesn't matter. } \\
\text { R2: I worry about what people } \\
\text { think of me even when I know it } \\
\text { makes no difference. }\end{array}$ & $\begin{array}{l}\text { Preocupo-me com o que as pessoas } \\
\text { pensarão de mim mesmo quando } \\
\text { sei que isso não faz nenhuma } \\
\text { diferença. }\end{array}$ \\
\hline $\begin{array}{l}\text { 3. I become tense and jittery if I } \\
\text { know someone is sizing me up. }\end{array}$ & $\begin{array}{l}\text { T1: Eu fico tenso e nervoso } \\
\text { quando eu sei que alguém está me } \\
\text { avaliando } \\
\text { T2: Eu me torno tenso e nervoso se } \\
\text { eu sei que alguém está me avaliando. }\end{array}$ & $\begin{array}{l}\text { R1: I get tense and anxious when I } \\
\text { know someone is evaluating me. } \\
\text { R2: I become tense and nervous if } \\
\text { I know that someone is evaluating } \\
\text { me. }\end{array}$ & $\begin{array}{l}\text { Eu me torno tenso e inquieto } \\
\text { quando sei que alguém está me } \\
\text { avaliando. }\end{array}$ \\
\hline $\begin{array}{l}\text { 4. I am unconcerned even if I } \\
\text { know people are forming an } \\
\text { unfavorable impression of me.I }\end{array}$ & $\begin{array}{l}\text { T1: Eu sempre fico desconsertado } \\
\text { se percebo que pessoas estão formando } \\
\text { uma impressão desfavorável de mim. } \\
\text { T2: Eu fico despreocupado mesmo } \\
\text { quando eu sei que as pessoas estão } \\
\text { formando uma impressão desfavo- } \\
\text { rável sobre mim. }\end{array}$ & $\begin{array}{l}\text { R1: I get uncomfortable if I noticed } \\
\text { people are having a bad impression } \\
\text { of me. } \\
\text { R2: I am worried even when I know } \\
\text { people are forming a bad impression } \\
\text { on me. }\end{array}$ & $\begin{array}{l}\text { Eu fico despreocupado mesmo se } \\
\text { eu sei que as pessoas estão formando } \\
\text { uma impressão desfavorável de } \\
\text { mim. }\end{array}$ \\
\hline
\end{tabular}


5. I feel very upset when I commit some social error.

6. The opinions that important people have of me cause me little concern.

7. I am often afraid that I may look ridiculous or make a fool of myself.

8. I react very little when other people disapprove of me.

9. I am frequently afraid of other people noticing my shortcomings.

10. The disapproval of others would have little effect on me.

11. If someone is evaluating me I tend to expect the worst.

12. I rarely worry about what kind of impression I am making on someone.

13. I am afraid that others will not approve of me.

14. I am afraid that people will find fault with me.

15. Other people's opinions of me do not bother me.

T1: Eu me sinto muito triste quando cometo um deslize/erro social. T2: Eu fico chateado quando cometo alguma gafe social.

T1: As opiniões que as pessoas que me são importantes têm a meu respeito causam-me pouca preocupação.

T2: As opiniões que pessoas importantes têm de mim me causam pouca preocupação.

T1: Eu frequentemente fico com medo de parecer ridículo ou fazer algo tolo.

T2: Eu frequentemente tenho medo de que possa parecer ridículo ou fazer papel de tolo.

T1: Eu reajo muito pouco quando outras pessoas me desaprovam. T2: Eu quase não tenho reação quando as pessoas me desaprovam.

T1: Frequentemente eu sinto medo de as pessoas perceberem meus defeitos.

T2: Eu frequentemente tenho medo de que as outras pessoas percebam meus defeitos.

T1: A desaprovação de outras pessoas teria um pequeno efeito sobre mim.

T2: A desaprovação vinda de outros teria um efeito pequeno sobre mim.

T1: Se alguém está me avaliando eu tenho a tendência de esperar o pior. T2: Se alguém está me avaliando, eu tendo a esperar o pior.

T1: Raramente eu me preocupo com o tipo de impressão que estou passando para alguém.

T2: Eu raramente me preocupo com o tipo de impressão que estou causando em alguém.

T1: Eu temo que os outros não me aprovem.

T2: Eu me preocupo que outros não me aprovarão.

T1: Eu temo que as pessoas detectem minhas falhas.

T2: Eu me preocupo que pessoas encontrarão defeitos em mim.

T1: A opinião dos outros a meu respeito não me incomodam. T2: As opiniões de outras pessoas sobre mim não me incomodam.
R1: I feel very sad when I make a social mistake.

R2: I get upset when I commit a social gaffe.

R1: The opinion of me of those I care about causes me a little worry. R2: The opinions that important people have of me cause me little concern.

R1: I frequently worry about look ridiculous or do something foolish. R2: I often am afraid that might seem ridiculous or make a fool role.

Frequentemente tenho medo de que possa parecer ridículo ou fazer

Eu me sinto muito chateado quando cometo alguma gafe.

As opiniões que pessoas importantes têm de mim me causam pouca preocupação.
R1: I don't react much when people disapprove me.

R2: I have almost no reaction when people I disapprove.

R1: Frequently I fear people notice my flaws.

R2: I often afraid that other people perceive my faults.

R1: The others disapproval would have a little effect over me. R2: The disapproval from the others would have a small effect on me.

$\mathrm{R} 1$ : If somebody is evaluating me I tend to expect the worst.

R2: If someone is evaluating me I tend to expect the worst.

R1: I rarely worry about what kind of impression I'm making to somebody.

R2: I rarely am concerned with the kind of impression that I am causing someone.

R1: I fear the others won't approve me.

R2: I am concerned that others will not approve me.

R1: I fear that people can detect my flaws.

R2: I am concerned that people will find faults in me.

R1: What the others think of me don't bother me.

R2: The views of other people about me did not bother me. papel de bobo.

Reajo minimamente quando outras pessoas me desaprovam.

Frequentemente tenho medo de que outras pessoas percebam meus defeitos.

A desaprovação dos outros teria pouco efeito em mim.

Se alguém está me avaliando, tendo a esperar o pior.

Raramente me preocupo com o tipo de impressão que estou causando em alguém.

Temo que outros não me aprovarão.

Temo que as pessoas encontrarão defeitos em mim.

As opiniões dos outros a meu respeito não me incomodam. 
16. I am not necessarily upset if I do not please someone.

17. When I am talking to someone, I worry about what they may be thinking about me.

18. I feel that you can't help making social errors sometimes, so why worry about it.

19. I am usually worried about what kind of impression I make.

20. I worry a lot about what my superiors think of me.

21. If I know someone is judging me, it has little effect on me.

22. I worry that others will think I am not worthwhile.

23. I worry very little about what others may think of me.

24. Sometimes I think I am too concerned with what other people think of me.

25. I often worry that I will say or do the wrong things.
T1: Eu não fico necessariamente triste caso eu não agrade alguém. T2: Eu não fico necessariamente chateado se eu não agrado a alguém.

T1: Quando eu estou falando com alguém, eu me preocupo com o que eles estão pensando sobre mim. T2: Quando eu estou conversando com alguém, eu me preocupo com o que possam estar pensando sobre mim.

T1: Eu acho que não tem como controlar algumas gafes sociais, então por que me preocupar com isso.

T2: Eu sinto que não posso evitar cometer gafes sociais às vezes, então não me preocupo com isso.

T1: Normalmente eu fico preocupado com a impressão que passo. T2: Eu fico frequentemente preocupado com o tipo de impressão que eu passo.

T1: Eu me preocupo muito com o que meus superiores pensam a meu respeito.

T2: Eu me preocupo muito com o que meus superiores acharão de mim.

T1: Se eu sei que tem alguém me julgando, isso tem pouco efeito sobre mim.

T2: Se eu sei que alguém está me julgando, isso tem pouco efeito sobre mim.

T1: Eu me preocupo se os outros me acharão desinteressante. T2: Eu me preocupo que outras pessoas me acharão sem valor.

T1: Eu me preocupo muito pouco sobre o que os outros podem pensar a meu respeito.

T2: Eu me preocupo muito pouco com o que outros pensarão de mim.

T1: Às vezes acho que estou muito preocupado com o que os outros pensam de mim.

T2: Às vezes eu acho que fico muito preocupado com o que as pessoas pensarão de mim.

T1: Eu frequentemente me preocupo se vou dizer ou fazer coisas erradas.

T2: Eu frequentemente me preocupo que eu fale ou faça coisas errado.
R1: I don't necessarily feel sad when I don't please somebody. R2: I am not necessarily upset if I do not please someone.

R1: When I talk to somebody, I worry about what they are thinking of me.

R2: When I am chatting with someone, I am concerned with what they may be thinking about me.

R1: I don't feel like we can control some social goofs, so I don't worry about it.

R2: I feel that I cannot avoid committing social gaffes sometimes, then I worry about it.

R1: Usually I get worried about the impression I'm causing. R2: I am often worried about the kind of impression I step.

R1: I worry too much about what my superiors think of me. R2: I am very worried about what my superiors will think about me.

$\mathrm{R} 1$ : If I'm aware somebody is judging me, that don't affect me much.

$\mathrm{R} 2$ : If I know someone is judging me, this has little effect on me.

R1: I worry if people think I'm not interesting.

R2: I am concerned that other people will find me no value.

R1: I care too little about what others may think of me. R2: I care very little about what others will think of me.

R1: Sometimes I think I'm too worried about what others think of me.

R2: Sometimes I think I am very worried about what people will think of me.

R1: I usually worry if I'm going to do or say something inappropriate. $\mathrm{R} 2$ : I often am concerned that I speak or do things wrong.
Não fico necessariamente chateado se não agrado a alguém.

Quando estou conversando com alguém, me preocupo com o que possam estar pensando sobre mim.

Sinto que as vezes não posso evitar cometer gafes, então, por quê me preocupar com isso.

Fico frequentemente preocupado com o tipo de impressão que passo.

Preocupo-me muito com o que os meus superiores pensam de mim.

Se eu sei que alguém está me julgando, isso tem pouco efeito em mim.

Eu me preocupo que os outros pensem que eu não tenho valor.

Eu me preocupo muito pouco com o que os outros possam pensar de mim.

Às vezes, acho que estou muito preocupado com o que os outros possam pensar de mim.

Frequentemente me preocupo que direi ou farei coisas erradas. 
26. I am often indifferent to the opinions others have of me.

27. I am usually confident that others will have a favorable impression of me.

28. I often worry that people who are important to me won't think very much of me.

29. I brood about the opinions my friends have about me.

30. I become tense and jittery if I know I am being judged by my superiors.
T1: Normalmente sou indiferente às opiniões que outros têm de mim. T2: Eu fico frequentemente indiferente com as opiniões de outros sobre mim

T1: Geralmente fico confiante que os outros terão uma impressão favorável de mim.

T2: Eu sou frequentemente confiante em que outros terão uma opinião favorável sobre mim.

T1: Eu frequentemente me preocupo que as pessoas que me são importantes não pensarão muito em mim.

T2: Eu frequentemente me preocupo que as pessoas que são importantes para mim não pensarão muito sobre mim.

T1: Eu fico ruminando sobre as opiniões que meus amigos têm sobre mim.

T2: Eu rumino sobre as opiniões que meus amigos têm de mim.

T1: Eu fico tenso e nervoso se eu sei que estou sendo julgado por meus superiores.

T2: Eu me torno tenso e nervoso se eu sei que estou sendo julgado por meus superiores.
R1: Usually I'm indifferent to what others think of me.

R2: I am often indifferent to the opinions of others about me.

R1: Generally, I get confident the others will have a good impression of me.

R2: I am frequently confident that others will have a favorable opinion about me.

R1: I normally worry about what those I care about will think of me. R2: I often am concerned that people who are important to me.

$\mathrm{R} 1$ : I keep ruminating what my friends think of me.

R2: I rumin on the views that my friends have of me.

R1: I get tense and anxious if I'm aware my superiors are judging me. R2: I become tense and nervous if I know I am being judged by my superiors.
Frequentemente sou indiferente às opiniões que os outros têm de mim.

Frequentemente fico confiante que os outros terão uma impressão favorável de mim.

Eu frequentemente me preocupo que as pessoas que me são importantes não pensarão muito em mim.

Fico ruminando sobre as opiniões que meus amigos têm de mim.

Eu me torno tenso e inquieto se sei que estou sendo julgado por meus superiores.
Alguns itens foram formados pela combinação das traduções realizadas por T1 e T2, enquanto, em alguns casos, foi privilegiada a sugestão de um deles. Ainda, houve situações em que os especialistas introduziram ligeiras alterações no sugerido pelos tradutores, de forma a manter a pertinência do construto avaliado.

Os itens $1,8,9,10,11,12,19,21,25$ e 29 tiveram traduções muito semelhantes por parte de T1 e de T2, obtendo-se, nesses casos, uma versão combinada de ambas. Nos itens 4 , 6, 20 e 23 também foi utilizada uma versão sintética de T1 e T2, mas levemente ajustada para obter maior conformidade com o padrão linguístico original.

No item 1, a diferença entre T1 e T2 foi apenas quanto ao uso de "se pareço" ou "em parecer". Optou-se por “em parecer", sendo suprimido o pronome "eu", utilizado por ambos os tradutores e de acordo com o item original, devido ao fato de seu uso não ser comum no discurso habitual da língua alvo.

No item 8, as propostas "reajo muito pouco" de T1 e "quase não tenho reação" de T2 foram convertidas em "reajo minimamente" na versão final. A última parte do enunciado foi comum aos dois tradutores e, portanto, mantida.

As propostas de T1 e T2 para o item 9 foram muito semelhantes, havendo apenas diferença entre "sinto medo" e "tenho medo". Optou-se pelo uso de "tenho medo".
No item 10, os termos propostos "teria um pequeno efeito" e "teria um efeito pequeno" foram adaptados para "teria pouco efeito" de modo a parecer mais natural. O mesmo ocorreu com "desaprovação de outras pessoas" e "desaprovação vinda dos outros", que foram levemente modificadas para "desaprovação dos outros", preservando-se, entretanto, o sentido original.

Apesar de T1 e T2 apresentarem enunciados bem semelhantes para o item 11, o primeiro tradutor utilizou "eu tenho a tendência", enquanto o segundo usou "eu tendo". A opção de T2 prevaleceu, nos parecendo uma correspondência mais adequada ao tempo verbal expresso na versão original do item.

Novamente as traduções foram bastante semelhantes no item 12, sendo a única diferença o uso de "passando para" por T1 e de "causando em" por T2. Ambas são corretas e adequadas; no entanto, como percebemos que na língua para a qual a escala estava sendo adaptada, o uso da expressão "causando em alguém" é mais frequente, optamos por adotar essa versão.

No item 19, o termo "usually", que em tradução literal seria "habitualmente", foi traduzido como "frequentemente" por T2 e como "normalmente" por T1. Foi selecionada a proposta de T2 por parecer mais próxima do sentido original. O termo "tipo de", mantido por T2 e não utilizado por T1, também foi preservado. 
Semelhante situação ocorreu no item 27, onde "usually" foi traduzido como "geralmente" por T1 e como "frequentemente" por T2. A conduta adotada foi a mesma, optandose por "frequentemente". O restante da oração foi utilizado conforme a tradução de T1, aproveitando-se "impressão" e não "opinião", como em T2, para representar o termo "impression".

As traduções para o item 21 foram praticamente idênticas, exceto pelo fato de que T1 usou "tem alguém" enquanto T2 optou por "alguém está", sendo mantido o sentido em ambas as propostas. No item final, optou-se por "alguém está".

A diferença entre $\mathrm{T} 1$ e $\mathrm{T} 2$ para o item 25 foi basicamente em relação ao tempo verbal utilizado. Buscando-se a correta adequação ao original, para "will say or do", utilizou-se "que direi ou farei".

No item 29, a expressão "brood about", que poderia ser traduzida literalmente como "meditar sobre", foi vertida para a língua alvo como "ruminar" por ambos os tradutores. Optando por respeitar o termo escolhido pelos tradutores, o tempo verbal mais adequado seria o utilizado por T2, "eu rumino"; no entanto, culturalmente, nota-se com mais frequência o uso da mesma expressão com o tempo verbal alterado, como proposto por T1: "fico ruminando". Devido à proposta de adaptação semântica, foi utilizada a versão de T1 na formação do item final.

Para o item 4, T1 optou pela utilização de "desconcertado" para a palavra "unconcerned", que significa "despreocupado" em uma tradução literal. Foi utilizada então a proposta de T2, sendo modificado, no entanto, o termo "sobre mim" para "de mim", conforme T1.

No item 6, notamos o uso de "pessoas que me são importantes" por T1 para "important people", o que seria uma alteração importante do original, uma vez que nele não notamos a referência de importância das pessoas em relação ao próprio sujeito. Permaneceu, então, a proposta de T2 "pessoas importantes", condizente com o enunciado original.

No item 20, notamos o uso de "pensam a meu respeito" por T1 e de "acharão de mim" por T2. Optou-se por utilizar uma versão combinada: "pensam de mim". O início do enunciado sofreu ligeira alteração de "eu me preocupo" para "preocupo-me" para ajustar-se aos padrões linguísticos.

Para o item 23 prevaleceu a proposta de T2, mostrando-se mais adequada, sendo, no entanto, realizado ajuste no tempo verbal de "pensarão" para "possam pensar".

No item 26 são utilizados dois termos diferentes para "often", que na tradução literal seria "frequentemente", conforme o proposto por T2 com "eu fico frequentemente"; no entanto, o "eu fico" mostrou-se pouco apropriado. Nesse sentido, manteve-se o estilo sugerido por T1 com seu "normalmente sou", substituindo-se o primeiro termo por "frequentemente", e assim formando a versão final do item.

Nos itens 15 e 28 , optou-se pela formulação sugerida por $\mathrm{T} 1$, e nos itens 2, 16, e 17 foram selecionadas as versões propostas por T2. Para o item 15, a diferença entre os tradutores foi apenas no uso de "dos outros" e "de outras pessoas".
Foi privilegiado o uso do primeiro. Já no item 28, T1 utilizou "muito em mim", o que se mostrou mais adequado a "much of me" do que o "muito sobre mim" proposto por T2.

O tempo verbal foi determinante para a escolha da versão de T2 para a representação do item 2 , sendo utilizado "pensarão" para "will think" em detrimento do "pensam" sugerido por T1. O termo "não faz a menor diferença" foi ajustado para "não faz nenhuma diferença" para obter maior familiaridade com o uso habitual na língua alvo.

No item 16, o uso de "chateado" mostrou-se mais apropriado ao "upset" do item original do que o "triste" sugerido por T1. A proposta de T2 foi mantida, sendo apenas suprimido o pronome "eu" de "se eu não agrado a alguém" por mostrar-se desnecessário.

Notou-se no item 17 maior adequação da expressão "possam estar pensando", de T2, para "may be thinking". O uso de "conversando", e não "falando", como sugerido por T1 para "talking", também foi um elemento para a escolha da versão de T2.

No item 5 foi priorizada a versão de T2, modificando-se apenas "fico" para "sinto", de acordo com o proposto por T1. Em relação à expressão "social error", apesar de entender que a tradução literal seria "erro social", conforme o proposto por T1, optou-se pelo termo "gafe", usado por T2, comum no português do Brasil, sem o termo "social" em seguida. O mesmo ajuste foi realizado no item 18 .

Nos itens 3 e 30, ambas as traduções foram semelhantes ao utilizar o termo "nervoso" como equivalência de "jittery". No entanto, ao verificar a pertinência do termo para a área de saúde mental, optou-se pela sua substituição por "inquieto", sendo maior a pertinência deste último, além de também não comprometer o sentido da formulação proposta na versão original.

Ainda em relação a esses dois itens, o uso da expressão "I become", a tradução por "eu me torno" proposta por T2 foi escolhida em detrimento do "eu fico" sugerido por T1 por estar mais próxima de uma tradução literal.

Para o item 7, as duas traduções são muito semelhantes. Foi preservada a de T2, apenas substituindo-se "tolo" por "bobo", relativos à expressão "make a fool of myself", por ser mais frequente na linguagem coloquial utilizada na cultura da população alvo para a utilização da escala.

Foi escolhido o termo "medo" para "afraid" em detrimento do "me preocupo" sugerido por T2 no caso dos itens 13 e 14. No item 22, é realizada uma versão sintética, mas priorizado o "sem valor" sugerido por T2 por se apresentar mais próximo do proposto no original. Finalmente, no item 24, é adotada a proposta de T1. Nesse caso, optou-se por manter o "estou" em vez de "sou", privilegiando a ênfase no momento atual.

Uma vez realizada a elaboração da versão sintética dos itens que formariam a primeira versão da escala em português do Brasil, partiu-se para a aplicação experimental do instrumento para investigação quanto à real adequação dos itens formulados à população à qual o instrumento se destinava. 
Para isso, contou-se com a colaboração de sujeitos voluntários, de diferentes níveis de escolaridade, diversas áreas de conhecimento e representativos de ambos os sexos. Esses participantes foram testados mediante o uso do MINI para descartar presença de transtornos psiquiátricos. O procedimento eliminou três dos 30 voluntários iniciais, participando efetivamente da análise 27 pessoas, tendo o grupo o seguinte perfil: 19 participantes do sexo feminino e oito do sexo masculino; sete sujeitos com nível fundamental, cinco com nível médio e 15 com nível superior, sendo estes últimos representantes das áreas humanas, biológicas e exatas.

Após responderem à FNE em aplicação experimental seguindo a padronização de aplicação conforme determinado no instrumento original, os sujeitos participaram de uma discussão quanto aos aspectos linguísticos do material utilizado.

Nessa discussão foram seguidas duas etapas. Na primeira, foi solicitado que o participante descrevesse com suas palavras o que entendia do enunciado contido na versão preliminar do instrumento. Isso foi realizado para todos os itens. $\mathrm{Na}$ segunda fase, foram solicitadas sugestões de termos alternativos que pudessem facilitar o entendimento do item.

As aplicações foram realizadas com os participantes com escolaridade de nível fundamental primeiro, depois com os de nível médio e, finalmente, concluindo o processo, com os que possuíam nível superior completo ou incompleto; neste último caso, o participante tinha concluído ao menos $50 \%$ do curso de graduação.

No grupo com ensino fundamental, notou-se omissão de resposta em três itens por parte de três participantes. $\mathrm{O}$ item 4 foi omitido por dois sujeitos, assim como o 27; eles explicaram ter omitido as respostas não entender o que significaria a palavra "favorável". No caso dos itens 5 e 18, verificouse a mesma omissão, pelo mesmo motivo mas em relação à palavra "gafe", por outro participante. Quanto a esses dois últimos itens citados, outros três participantes, na posterior discussão, referiram ter tido dificuldade no entendimento do termo empregado.

Em relação à solicitação de descrição pelos participantes do entendimento dos enunciados, de modo geral as definições fornecidas conseguiram chegar ao sentido proposto pelo instrumento, mostrando que ele estava sendo bem compreendido. Constituíram exceções os itens 5 e 18, devido à dificuldade de compreensão já citada da palavra "gafe", e também o item 29, uma vez que os participantes se mostraram confusos quanto ao significado do termo "ruminante".

O item 28 gerou leve confusão em relação ao "que me são". Ainda assim, após breve reflexão sobre o enunciado e sem interferência do pesquisador, a questão ficou clara para os participantes. A questão 2 também consumiu um tempo um pouco maior para o entendimento.

Esse grupo não forneceu sugestões diretas de termos alternativos, mantendo-se mais reservado e passivo do que os grupos representativos dos outros níveis de escolaridade. No entanto, no item 8, um participante colocou que "pouco" seria mais "comum" do que o "minimamente" empregado, ao que outro participante então mencionou ter tido que ler duas vezes a questão, durante a aplicação, para entender bem o que "queria dizer".

Entre os sujeitos que apresentavam nível médio, encontramos apenas uma omissão, relativa ao item 29 , na aplicação experimental, justificada posteriormente pelo respondente como devida à não compreensão do termo "ruminando".

Ao ser solicitado esclarecimento do sentido dos enunciados aos participantes desse grupo, todos se saíram bem, sendo, no entanto, notada alguma confusão em relação ao uso da palavra "gafe" por um dos respondentes, que revelou ter marcado a questão ao acaso apenas para não deixá-la sem resposta. O mesmo ocorreu com o termo "ruminante".

Esse grupo sinalizou que o item 27 não parecia "natural", mas não souberam esclarecer o motivo da sua impressão. No que se refere à palavra "gafe", um dos participantes, durante o processo, explicou a outro sujeito que mencionava não compreendê-la bem que a palavra seria equivalente a "dar vexame", "pagar mico" ou "passar vergonha", tendo a concordância de outro dos sujeitos quanto a suas colocações.

Dada a dificuldade com o termo "favorável" no grupo de nível fundamental, optou-se por questionar diretamente ao novo grupo quanto ao seu entendimento do significado da palavra. Os sujeitos mostraram correta compreensão, restringindo-se, então, o problema com esse vocábulo apenas aos participantes com escolaridade mais baixa.

Os integrantes deste segundo grupo mostraram-se mais participativos no processo e ofereceram algumas sugestões, como a substituição do termo "minimamente" do item 8 por "pouco", de forma a ficar mais próximo da linguagem utilizada por eles no cotidiano, e a modificação do uso de "sobre mim" para "de mim" no item 17.

Finalizada a análise do material fornecido por esse grupo, passou-se à aplicação experimental com o grupo de nível superior, sendo esse formado por profissionais graduandos e já graduados nas áreas humanas, exatas e biológica. Dos participantes desse grupo, cinco tinham conhecimentos relativos à área de saúde mental, mas não conheciam a escala em estudo.

Não houve omissões nos protocolos desses participantes, e todos mostraram perfeito entendimento do sentido dos enunciados quando questionados sobre isso. Foi o grupo que mais participou oferecendo sugestões para "melhorias" do instrumento.

No item 4, foi sugerida a remoção do pronome "eu", antes de "sei que", considerado pelo grupo como "desnecessário". Para o item 14, sugeriram substituir "as pessoas" por "os outros" para que ficasse de acordo, segundo eles, com a estrutura usada nos itens 22,23 e 24 . Ainda em relação a esses mesmos itens, acrescentaram que o uso de "os outros" neles seria "desnecessário", podendo ser suprimido.

Para o item 25, questionou-se a possibilidade de alteração do "que direi ou farei" para "se direi ou farei". Em relação ao item 27 , foi defendido que "fico confiante" deveria ser alterado para "confio". 
O grupo foi ainda questionado sobre termos que geraram dificuldades nas aplicações dos grupos anteriores como "gafe", "favorável" e "ruminando", sendo que entre eles os termos não causaram confusão.

Foi sugerida como alternativa para "impressão desfavorável" o uso de "má impressão" ou "impressão ruim". Para "gafe", foi proposto o termo "vexame", já sugerido anteriormente pelo grupo de nível médio de escolaridade, sendo, porém, acrescido de "em público", já que o termo "gafe" se refere a um acontecimento que ocorre socialmente.

Finalmente, para o vocábulo "ruminante" (em "fico ruminando"), as sugestões foram "fico pensando", "fico meditando" e "fico refletindo". Um dos participantes sugeriu ainda acrescentar um "sem parar" ao "pensando", pois, segundo ele, "pensando sem parar" refletiria a "natureza da ruminação", ou seja, "pensamentos que vão e voltam". Outros sujeitos concordaram com ele e, ainda, uma participante forneceu a opção de uso de "pensando continuamente".

Com as aplicações experimentais concluídas, foi realizada a síntese do material coletado para a elaboração final dos enunciados. Os itens 1, 2, 3, 6, 7, 9, 10, 11, 12, 13, 15, $16,19,20,21,26,28$ e 30 permaneceram sem alterações na versão final da escala.

Os itens 14 e 17, apesar das sugestões realizadas, foram mantidos conforme a proposta inicial, já que foram bem entendidos por todos os grupos, no intuito de manter a máxima semelhança com o instrumento original. O mesmo ocorreu em relação aos itens 22,23 e 24 , nos quais foi mantida a expressão "os outros".

No item 25, havendo dificuldade em um dos grupos, e sendo a mudança sugerida não prejudicial ao sentido proposto inicialmente pelo instrumento, optou-se pela modificação de "que" por "se", buscando melhor entendimento do item pelos respondentes. Com o mesmo intuito, nos itens 4 e 27, a expressão "impressão favorável" foi substituída por "boa impressão".

A palavra "gafe", que gerou dificuldade nos itens 5 e 18, foi modificada para "vexame em público", conforme sugestão dos participantes, preservando o significado original e tornando-se mais clara à compreensão.

No item 8 , no qual foi questionado o uso de "minimamente", fornecendo-se como opção "pouco" pelos participantes da aplicação experimental, os autores optaram pela alteração para "muito pouco", sendo semelhante ao sugerido e, ao mesmo tempo, mantendo a pertinência em relação ao que consta no item original, "very little".

O uso de "fico confiante", no item 27, também foi abordado pelos sujeitos, sendo sugerido o uso alternativo de "confio". Essa alteração, no entanto, provocaria uma modificação no sentido da frase, ao sugerir uma postura mais ativa do que passiva, contrária, então, ao apresentado originalmente. Devido a esse fator, foi mantido o uso de "fico confiante". Quanto ao "favorável", foi feita alteração semelhante à realizada no item 4.

Finalmente, no item 29, tivemos dificuldade com o uso da expressão original "I brood about", traduzida como "Fico ruminando". A questão do tempo verbal já havia sido apontada como um problema na construção da versão sintética, sendo, então, realizada alteração para adequação ao uso na língua alvo. Já o uso de "ruminando" gerou dificuldades e mostrou-se não adequado durante a aplicação experimental, ficando clara a necessidade de sua substituição.

Nesse sentido, dentre as propostas de termos alternativos sugeridas pelos sujeitos, a que mais se aproximaria do sentido original seria "fico meditando". No entanto, a fim de manter a coerência com a cultura à qual é destinado o uso da escala adaptada, acreditamos que o uso de "meditando" não evocaria o sentido de pensamento cíclico e repetitivo implícito no termo "ruminando", escolhido por ambos os tradutores convidados. Assim, para reduzir esse problema, optamos pelo uso de "penso continuamente".

Temos, então, os 30 itens em sua versão final, que constitui a versão da FNE para o português do Brasil.

\section{Discussão}

É clara a importância do uso de instrumentos estruturados para a área de psiquiatria e saúde mental com boas propriedades psicométricas que garantam, para além da precisão, que seus achados, de fato, mensurem aquilo que pretendem medir.

Isso ocorre, em parte, devido a que essa área de conhecimento ainda não possui recursos tais como testes clínicos ou marcadores biológicos que sejam capazes de fornecer bases indiscutíveis para uma correta identificação dos transtornos mentais. Mesmo havendo disponibilidade desse tipo de recurso diagnóstico, ainda assim teríamos nos instrumentos de medida um recurso rápido e de baixo custo, o que deve ser considerado principalmente quando pensamos em termos de atuação em serviços que contam com pouca verba.

A correta aferição de sinais e sintomas, por sua vez, não pode ser deixada de lado, considerando que a não identificação de quadros de saúde mental pode acarretar graves prejuízos para a vida dos sujeitos. Um correto diagnóstico propicia a possibilidade de tratamento ainda nas fases iniciais de desenvolvimento do transtorno, prevenindo o aparecimento de comorbidades e evitando que, além dos danos diretos que podem causar a seus portadores, esses transtornos atuem como fatores de risco ou de contribuição para maior morbidade e mortalidade também em quadros de adoecimento orgânico.

$\mathrm{O}$ uso de instrumentos de medida transcende ainda a área de avaliação para fins puramente diagnósticos e se insere no contexto dos atendimentos clínicos, seja para avaliar o progresso do paciente ao longo do tratamento ou mesmo a eficácia dos procedimentos adotados, seja como auxiliar no processo psicoeducativo.

Com a finalidade de elaboração da tradução e adaptação semântica da FNE, os itens originais da FNE foram encaminhados a tradutores independentes e sem conhecimentos prévios sobre a escala. Posteriormente, o material derivado 
dessas traduções foi encaminhado para a realização de retrotraduções por dois outros profissionais.

A opção por trabalhar com duas traduções e duas retrotraduções mostrou-se proveitosa, pois permitiu ampla discussão quando houve divergência entre as versões fornecidas, possibilitando, desse modo, uma maior adequação dos enunciados à proposta original da escala.

A familiaridade dos tradutores e avaliadores com a área de saúde mental facilitou o trabalho de adaptação semântica, pois apesar do desconhecimento prévio quanto ao instrumento específico, seus conhecimentos acerca do construto avaliado permitiram ajustes finos nos termos escolhidos para representar os itens originais.

A aplicação experimental possibilitou um ajuste final da versão sintética de forma que ela se apresentasse inteligível para aqueles que possuem nível de escolaridade fundamental e não comprometesse o interesse daqueles que têm mais anos de escolaridade, evitando comprometimento da validade aparente.

Com base nos dados obtidos nesse procedimento, foram então realizados os últimos ajustes no instrumento, formando assim sua versão final para o português. Estudos posteriores deverão ser realizados para avaliação de validade, fidedignidade e estabelecimento de normas para a população brasileira.

\section{Referências}

1. Watson D, Friend R. Measurement of social-evaluative anxiety. J Consult Clin Psychol. 1969;33(4):448-57.

2. Levitan MN, Nascimeno I, Freire RC, Mezzasalma MA, Nardi AE. Equivalência semântica da versão brasileira da Social Avoidance and Distress Scale (SADS). Rev Psiquiatr RS. 2008;30(1):49-58.

3. Versiani M. Princípios gerais básicos das escalas de avaliação. In: Centro de Pesquisa em Psicobiologia Clínica, editor. Escalas de avaliação para monitorização de tratamentos com psicofármacos. São Paulo: Associação Fundo de Incentivo à Psicofarmacologia; 1989. p. 1-20.

4. Goldner EM, Bilsker D. Evidence-based psychiatry. Can J Psychiatry. 1995;40(2):97-101.

5. Geddes J, Carney S. Recent advances in evidence-based psychiatry. Can J Psychiatry. 2001;46(5):403-6.

6. Milne D, Keegan D, Paxton R, Seth K. Is the practice of psychological therapists evidence-based? Int J Health Care Qual Assur Inc Leadersh Health Serv. 2000;13(1):8-14
7. Del Prette ZA, Del Prette A. Inventário de habilidades sociais: manual de aplicação, apuração e interpretação. São Paulo: Casa do Psicólogo; 2001.

8. Nunes CH, Hutz CS. Escala Fatorial de Socialização. São Paulo: Casa do Psicólogo; 2007.

9. Berger W, Mendlowicz MV, Souza WF, Figueira I. Equivalência semântica da versão em português da Post-Traumatic Stress Disorder Checklist-Civilian Version (PCL-C) para rastreamento do transtorno de estresse pós-traumático. Rev Psiquiatr RS. 2004;26(2):167-75.

10. Scagliusi FB, Cordás TA, Polacow VO, Coelho D, Alvarenga M, Philippi ST, et al. Tradução da escala de desejo de aceitação social de Marlowe \& Crowne para a língua portuguesa. Rev Psiq Clin. 2004;31(6):272-8.

11. Picon P, Gauer GJ, Fachel JM, Manfro GG. Desenvolvimento da versão em português do Social Phobia and Anxiety Inventory (SPAI). Rev Psiquiat RS. 2005;27(1):40-50.

12. Vilete L, Figueira I, Coutinho E. Adaptação transcultural para o português do Social Phobia Inventory (SPIN) para utilização entre estudantes adolescentes. Rev Psiquiatr RS. 2006;28(1):40-8.

13. Cunha JA. Manual da versão em português das escalas Beck. São Paulo: Casa do Psicólogo; 2001.

14. Spielberger CD (1927). Manual do Inventário de Expressão da Ansiedade como Estado e Traço (STAXI) (traduzido por Biaggio AM). $2^{\mathrm{a}}$ ed. São Paulo: Vetor; 2003.

15. Gauer GJ, Picon P, Vasconcellos SJ, Turner SM, Beidel DC. Validation of the Social Phobia and Anxiety Inventory for Children (SPAI-C) in a sample of Brazilian children. Braz J Med Biol Res. 2005;38(5):795-800.

16. de Lima Osório F, Crippa JA, Loureiro SR. A study of the discriminative validity of a screening tool (MINI-SPIN) for social anxiety disorder applied to Brazilian university students. Eur Psychiatry. 2007;22(4):239-43.

17. von Diemen LV, Szobot CM, Kessler F, Pechansky F. Adaptation and construct validation of the Barratt Impulsiveness Scale (BIS 11) to Brazilian Portuguese for use in adolescents. Rev Bras Psiquiatr. 2007;29(2):153-6.

18. Lima LA, Gonçalves S, Lovisi G, Pereira BB. Validação transcultural da Escala de Avaliação de Limitações do Comportamento Social - SBS-BR. Rev Psiquiatr Clin. 2003;30(4):126-38.

19. American Psychiatric Association. Manual Diagnóstico e Estatístico dos Transtornos Mentais: DSM-IV-TR. Porto Alegre: Artmed; 2003.

20. Herdman M, Fox-Rushby J, Badia X. A model of equivalence in the cultural adaptation of HRQoL instruments: the universalist approach. Qual Life Res. 1998;7(4):323-35.

21. Brislin RW. Back-translation for cross-cultural research. J Cross Cult Psychol. 1970;1(3):185-216.

22. Werner O, Campbell D. Translating, working though interpreters and the problem of decentering. In: Naroll R, Cohen R, editors. A handbook of method in cultural anthropology. New York: American Museum of Natural History; 1970. p. 398-420.

23. Pasquali L. Psicometria: teoria dos testes na psicologia e na educação. Petrópolis: Vozes; 2003.

24. Osório FL, Crippa JA, Loureiro, SR. Instrumentos de avaliação do transtorno de ansiedade social. Rev Psiquiatr Clin. 2005;32(2):73-83.

25. Sheehan DV, Lecrubier Y, Sheehan KH, Amorim P, Janavs J, Weiller E, et al. The Mini International Neuropsychiatric Interview (MINI): the development and validation of a structured diagnostic psychiatric interview for DSM-IV and ICD10. J Clin Psychiatry. 1998;59 Suppl 20:22-33. 


\section{Anexo}

\section{Escala de Medo de Avaliação Negativa}

Para as seguintes afirmativas, por favor, responda se são verdadeiras ou falsas para você. Circule $V$ para verdadeiro e $F$ para falso.

1. Raramente me preocupo em parecer tolo aos outros.

2. Preocupo-me com o que as pessoas pensarão de mim mesmo quando sei que isso não faz nenhuma diferença

3. Eu me torno tenso e inquieto quando sei que alguém está me avaliando.

4. Eu fico despreocupado mesmo se eu sei que as pessoas estão formando uma impressão ruim de mim.

5. Eu me sinto muito chateado quando cometo algum vexame em público.

6. As opiniões que pessoas importantes têm de mim me causam pouca preocupação.

7. Frequentemente tenho medo de que possa parecer ridículo ou fazer papel de bobo.

8. Reajo muito pouco quando outras pessoas me desaprovam.

9. Frequentemente tenho medo de que outras pessoas percebam meus defeitos.

10. A desaprovação dos outros teria pouco efeito em mim.

11. Se alguém está me avaliando, tendo a esperar o pior.

12. Raramente me preocupo com o tipo de impressão que estou causando em alguém.

13. Temo que outros não me aprovarão.

14. Temo que as pessoas encontrarão defeitos em mim.

15. As opiniões dos outros a meu respeito não me incomodam.

16. Não fico necessariamente chateado se não agrado a alguém.

17. Quando estou conversando com alguém, me preocupo com o que possam estar pensando sobre mim.

18. Sinto que às vezes não posso evitar cometer vexame em público, então, por quê me preocupar com isso.

19. Fico frequentemente preocupado com o tipo de impressão que passo.

20. Preocupo-me muito com o que os meus superiores pensam de mim.

21. Se eu sei que alguém está me julgando, isso tem pouco efeito em mim.

22. Eu me preocupo que os outros pensem que eu não tenho valor.

23. Eu me preocupo muito pouco com o que os outros possam pensar de mim.

24. Às vezes, acho que estou muito preocupado com o que os outros possam pensar de mim.

25. Frequentemente me preocupo se direi ou farei coisas erradas.

26. Frequentemente sou indiferente às opiniões que os outros têm de mim.

27. Frequentemente fico confiante que os outros terão uma boa impressão de mim.

28. Eu frequentemente me preocupo que as pessoas que me são importantes não pensarão muito em mim.

29. Penso continuamente sobre as opiniões que meus amigos têm de mim.

30. Eu me torno tenso e inquieto se sei que estou sendo julgado por meus superiores. 